\title{
黑盖木层孔菌发酵菌丝体和发酵液中活性多糖的 结构鉴定
}

李霞，焦丽丽，张旭，田文敏，陈珊，张丽萍"

东北师范大学生命科学学院，长春 130024

* 联系人, E-mail: zhanglp625@163.com

收稿日期: 2008-01-21；接受日期: 2008-01-25

\begin{abstract}
摘要黑盖木层孔菌经液体发酵培养后, 分别从菌丝体和发酵液中分离 纯化得到水溶性多糖PNW1 和PNM1. PNW1 和PNM1 均能抑制小鼠体内移 植性肉瘤Sarcoma 180 的生长. 在 $400 \mathrm{mg} / \mathrm{kg}$ 剂量时, 连续灌胃 10 天, 菌丝体 多糖PNW1 的抑瘤率可达 $74.70 \%$, 优于发酵液多糖PNM1(55.84\%). 两种多 糖平均分子量分别为 33 和 $29 \mathrm{kD}$. 气相色谱分析表明, 两种多糖均由葡萄 糖、半乳糖、甘露糖、阿拉伯糖和岩藻糖组成. 经部分酸水解、高碘酸氧化、 Smith 降解、 ${ }^{13} \mathrm{C}$ NMR、甲基化及其产物的 GC-MS分析, 确定了 PNW1 和 PNM1 的结构.
\end{abstract}

\section{关键词}

黑盖木层孔菌(Phellinus nigricans) 多糖

化学结构
多糖和糖蛋白复合物广泛存在于真菌、酵母、藻 类和高等植物中. 由于多糖具有免疫药理活性 ${ }^{[1]}$, 近 些年来被广泛研究. 多糖的活性与其单糖组成, 糖苷 键的构型和连接方式，取代基团的种类、位置和数量 等结构特点有着密切的关系. 一些活性多糖的结构 已经被报道 ${ }^{[2 \sim 7]}$.

黑盖木层孔菌(Phellinus nigricans)属担子菌. 与 之同属的裂缝木层孔菌(Phellinus rimosus)、鲍姆木层 孔菌(Phellinus baumii)、裂蹄木层孔菌(Phellinus linteus) 和淡黄木层孔菌(Phellinus gilvus)等菌种的生物活性和 结构特点已有研究 ${ }^{[4,8 \sim 10]}$. 其中, 对裂蹄木层孔菌的研 究较多, 它能够调节细胞免疫和体液免疫 ${ }^{[11]}$, 而其抗 肿瘤活性明显优于其他担子菌 ${ }^{[12,13]}$. 本实验材料黑盖 木层孔菌已经发酵培养成功, 初步研究表明其发酵液 多糖具有免疫和抗肿瘤活性. 黑盖木层孔菌多糖的结 构研究没有相关报道. 本文主要研究了黑盖木层孔菌 菌丝体多糖和发酵液多糖的结构特点.

\section{1 材料与方法}

\section{1 一般方法}

WZZ-T1 自动旋光仪测定比旋光度. 样品经 $\mathrm{KBr}$ 压片，在(400 4000)/cm 区间扫描红外吸收, SEPCORDIR 型红外光谱仪记录红外光谱. INOVA-500 核 磁共振仪检测 ${ }^{13} \mathrm{C}$ NMR. 高效排阻色谱法(HPSEC)检 测多糖平均分子量 ${ }^{[14]}$. 日本 SHIMADZU LC-10AT $\mathrm{VP}$, TSK-G3000PW $\mathrm{XL}_{\mathrm{XL}}$ 色谱柱 $(7.8 \mathrm{~mm} \times 30.0 \mathrm{~cm})$, SHIMADZU RID-10A监测器, 洗脱液 $0.7 \% \mathrm{Na}_{2} \mathrm{SO}_{4}$, 流速 $0.5 \mathrm{~mL} / \mathrm{min}$, 柱温 $40^{\circ} \mathrm{C}$. 采用已知分子量的标准葡聚 糖(标准分子量: $130,80,50,20,10$ )绘制标准曲线. 酚 一硫酸法测定多糖的总糖含量, $D$-葡萄糖绘制标准曲 线 ${ }^{[15]}$. 间羟联苯法测定糖醛酸的含量, $D$-葡萄糖醛酸 绘制标准曲线 ${ }^{[16]}$.

气相色谱 $(\mathrm{GC})$ 检测单糖组成. 多糖经 $2 \mathrm{~mol} / \mathrm{L}$ 三 氟乙酸 $(\mathrm{TFA}) 120^{\circ} \mathrm{C}$ 封管水解 $3 \mathrm{~h}$, 蒸干除去TFA, 经 
$\mathrm{KBH}_{4}$ 还原和乙酰化 $(1: 1$ 的无水吡啶和乙酸䣶, $\left.90^{\circ} \mathrm{C}, 1 \mathrm{~h}\right)^{[17,18]}$, 得到的乙酰化产物 $\mathrm{GC}$ 分析.

\section{2 多糖的制备}

黑盖木层孔菌子实体由吉林省生物研究所提供. 菌种经 $500 \mathrm{~mL}$ 雉形瓶发酵 7 天(培养温度 $28^{\circ} \mathrm{C}$, 转速 $140 \mathrm{r} / \mathrm{min}), 4000 \mathrm{r} / \mathrm{min}$ 离心 $15 \mathrm{~min}$, 收集菌丝体, 用蒸 馏水洗两次, 乙醇脱脂, 热水提取 $2 \mathrm{~h}$, 乙醇醇沉, 得 到菌丝体水溶性粗多糖, 命名为 PNW(菌丝体多糖).

发酵上清液浓缩, 乙醇醇沉, 得到发酵液提取物, 命名为 $\mathrm{PNM}$ (胞外多糖).

多糖溶于水后 $-20^{\circ} \mathrm{C}$ 冷冻 $24 \mathrm{~h}$, 融化至 $4^{\circ} \mathrm{C}, 4000$ $\mathrm{r} / \mathrm{min}$ 离心, 反复数次至无沉淀产生. 上清的糖溶液 经 DEAE-纤维素 $(3 \times 45 \mathrm{~cm})$, 蒸馏水洗脱, 流速 1 $\mathrm{mL} / \mathrm{min}$. 大部分色素被除掉, 水洗脱部分为中性糖. 再经 Separose CL-6B(2.5 cm × $90 \mathrm{~cm})$ 柱层析制备, $0.9 \% \mathrm{NaCl}$ 洗脱, 流速 $0.5 \mathrm{~mL} / \mathrm{min}$, 收集糖峰洗脱液, 透析后冻干, 分别命名为 PNW1 和 PNM1.

\section{3 部分酸水解}

$95^{\circ} \mathrm{C}, 0.05 \mathrm{~mol} / \mathrm{L}$ 三氟乙酸水解多糖 $2 \mathrm{~h}$. 降至室温 后, 离心(4000 r/min, $10 \mathrm{~min})$, 将沉淀冻干, 留做 $\mathrm{GC}$ 分 析. 上清用无水乙醇赶酸至中性( $\mathrm{pH}$ 6 7), 蒸馏水透析 $48 \mathrm{~h}$. 将袋外透析液冻干, GC 分析; 袋内液加 10 倍体 积无水乙醇, 醇沉过夜, 离心(4000 r/min, $10 \mathrm{~min})$, 沉 淀冻干，作 $\mathrm{GC}$ 分析; 上清冻干，留做 $\mathrm{GC}$ 分析.

\section{4 高碘酸氧化和 Smith 降解}

多糖 $25 \mathrm{mg}$, 用少量水溶解于 $25 \mathrm{~mL}$ 容量瓶中, 然后加入 $30 \mathrm{mmol} / \mathrm{L} \mathrm{NaIO}_{4} 12.5 \mathrm{~mL}$, 定容. 放置在暗 处反应(室温), 间隔时间 $(0,6,12,24,36,48,60 \cdots \cdots \cdot h)$ 取样 $0.1 \mathrm{~mL}$, 用蒸馏水稀释 250 倍, 以蒸馏水作空白 对照, 在 $223 \mathrm{~nm}$ 波长处测光密度值, 直到光密度值恒 定为止. 加乙二醇终止反应, 高碘酸氧化完成. 查标 准曲线, 计算出高碘酸的消耗量. 用 $\mathrm{NaOH}$ (用邻苯二 氢钾标定)滴定，计算得甲酸生成量.

将乙二醇处理后的溶液透析, 加入 $\mathrm{NaBH}_{4}$ 还原 过夜. 用 50\% HAc中和至pH 6 7, 透析. 取 $1 / 3$ 冻干 后做 GC分析, 剩余部分加入等体积的 $1 \mathrm{~mol} / \mathrm{L} \mathrm{H}_{2} \mathrm{SO}_{4}$, $25^{\circ} \mathrm{C}$ 水解 $40 \mathrm{~h}, \mathrm{BaCO}_{3}$ 中和至 $\mathrm{pH}$ 为 6 , 用定量滤纸过
滤, 滤液用蒸馏水透析, 袋外部分干燥做 $\mathrm{GC}$ 分析, 袋 内加乙醇醇析, 离心, 上清及沉淀部分冻干后分别做 $\mathrm{GC}$ 分析 ${ }^{[19]}$.

\section{5 甲基化分析}

彻底干燥糖样, 溶于无水二甲亚砜(DMSO, 以 $4 \mathrm{~A}$ 分子笁脱水), 按照文献[20]描述甲基化 3 次. IR 图谱检测在(3200 3700)/cm处无吸收峰, 即无羟基 吸收峰，则证明多糖甲基化完全。完全甲基化的多 糖分别经 $85 \%$ 甲酸和 $2 \mathrm{~mol} / \mathrm{L}$ 三氟乙酸水解, 然后甲 醇蒸除酸. 水解产物再经 $\mathrm{NaBH}_{4}$ 还原, 乙酰化(参 照 1.1 小节方法)后进行 GC-MS分析. HP-5 色谱柱 30 $\mathrm{m} \times 250 \mu \mathrm{m} \times 0.25 \mathrm{~nm}$, 程序升温 $5^{\circ} \mathrm{C} / \mathrm{min}$ 至 $280^{\circ} \mathrm{C}, \mathrm{He}$ 为载气.

\section{6 体内抗肿瘤实验}

雄性 BALB/c 小鼠, $8 \sim 12$ 周龄, 购自吉林大学病 理实验中心. 自由进食.

Sarcoma 180 细胞常规腹腔传代. BALB/c小鼠, 腹腔收集Sarcoma 180 细胞, PBS洗两次后重新悬浮成 细胞悬液 $\left(5 \times 10^{6}\right.$ 细胞 $\left./ \mathrm{mL}\right)$. 无菌条件下, $0.2 \mathrm{~mL}$ 的 PBS(含有 $10^{6}$ 个肿瘤细胞) 接种于实验小鼠的右前肢 腋皮下, 制备移植瘤模型. 将小鼠随机分组, 每组 9 只. 样品 $(400 \mathrm{mg} / \mathrm{kg}$ ) 和环林酰胺 $(25 \mathrm{mg} / \mathrm{kg})$ 分别溶于 生理盐水, 每天口服 $0.2 \mathrm{~mL}$, 连续给药 10 天后, 将小 鼠称重. 阴性对照组每天口服同体积的生理盐水.

抑瘤率 $=($ 阴性对照组瘤重-治疗组瘤重)/阴性对 照组瘤重 $\times 100 \%$

结果用平均值土标准差表示. 采用 $t$ 检验方法统 计分析, $P<0.05$ 差异为显著, $P<0.01$ 为极显著.

\section{2 结果}

\section{1 多糖的制备}

分别从菌丝体和发酵液中提取出粗多糖, 经 DEAE-cellulose和Sepharose CL-6B纯化得到 PNW1 和 PNM1 两种均一多糖. HPSEC上均呈单一对称峰, 经测定分子量分别为 33 和 $29 \mathrm{kD}$ (校正方程: $y=$ $\left.17.599-0.5757 x ; R^{2}=0.9837\right)$. 气相色谱分析单糖组成, 结果见表 1 . 两种多糖均由葡萄糖 (Glc)、半乳糖 (Gal)、甘露糖(Man)和少量的阿拉伯糖(Ara)、岩藻糖 
(Fuc)构成, 摩尔比分别为 $3.26 ８ .77 ： 6.44: 1.00$ : 1.35 (PNW1) 和 $20.06: 8.72: 6.94: 1.00: 0.76$ (PNM1). 均未检测出糖醛酸. 红外光谱显示, PNW1 和 PNM1 在(1000 1100)/cm, (1400 1530)/cm, (2800 $2900) / \mathrm{cm}$ 和(3100 3500)/cm均有多糖的特征吸收峰 (表 1).

表 1 PNW1 和 PNM1 的单糖组成、分子量和旋光值

\begin{tabular}{ccc}
\hline 样品 & PNW1 & PNM1 \\
\hline 平均分子量 $\mathrm{s}$ & $3.3 \times 10^{4}$ & $2.9 \times 10^{4}$ \\
{$[\alpha]_{\mathrm{D}}{ }^{20}$} & +63.8 & +41.3 \\
单糖组成 $/ \mathrm{mol}$ & & \\
葡萄糖 & 3.26 & 20.06 \\
半乳糖 & 8.77 & 8.72 \\
甘露糖 & 6.44 & 6.94 \\
阿拉伯糖 & 1.00 & 1.00 \\
岩藻糖 & 1.35 & 0.76 \\
\hline
\end{tabular}

PNW1 经高碘酸氧化, 消耗 $0.282 \mathrm{mmol}$ 高碘酸, 产生甲酸 $0.128 \mathrm{mmol}$. 高碘酸的消耗量超过甲酸生 成量的 2 倍，说明存在大量 $1 \rightarrow$ 或 $1 \rightarrow 6$ 键型. Smith 降解后产生大量的甘油, 没有检测出赤藓醇, 说明 PNW1 可能含有 $1 \rightarrow, 1 \rightarrow 6$ 或 $1 \rightarrow 2,6$ 键型, 但是不含 $1 \rightarrow 4$ 或 $1 \rightarrow 4,6$ 键型. Smith 降解中没有检测出糖残基, 说明 PNW1 糖苷键均可以被高碘酸氧化. 结合部分 酸水解和 Smith 降解的结果, 可知 Glc $1 \rightarrow 6$ 和 $\mathrm{Gal}$ $1 \rightarrow 6$ 共同构成骨架结构, 分支点为 Glc $1 \rightarrow 2,6$ 和 Gal $1 \rightarrow 2,6$. 支链主要为 Man $1 \rightarrow 6$.

\subsection{PNW1 的结构分析}

PNW1 部分酸水解后得到 4 个部分PNW1 $1^{\mathrm{a}}$, $P N W 1^{b}, P N W 1^{c}{ }^{\mathrm{c}}$ 和 $\mathrm{PNW} 1^{\mathrm{d}}, \mathrm{GC}$ 分析结果见表 2. $P N W 1^{\mathrm{a}}$ 和 $\mathrm{PNW} 1^{\mathrm{b}}$ 均含有葡萄糖和半乳糖, 它们构成 PNW1 的骨架结构. PNW $1^{\mathrm{c}}$ 和PNW $11^{\mathrm{d}}$ 中检出甘露糖、 阿拉伯糖和岩藻糖，它们位于PNW1 的侧链.

PNW1 的甲基乙酰化产物经 GC-MS，结果见图 1 和表 3. GC 共检测出 8 个峰, 分别为 2,3,5-三-O-甲 基-阿拉伯糖醇, 2,3,4-三-O-甲基-岩藻糖醇, 2,3,4,6-四 -O-甲基-甘露醇，2,3,4-三-O-甲基-葡萄糖醇，2,3,4-三 -O-甲基-甘露醇，2,3,4-三-O-甲基-半乳糖醇，3,4-二 -O-甲基-葡萄糖醇和 3,4-二-O-甲基-半乳糖醇。检测 出少量的阿拉伯糖醇和岩藻糖醇, 可能与二者在 PNW1 中的含量有关.

表 2 部分酸水解 GC 分析结果

\begin{tabular}{|c|c|c|c|c|c|}
\hline 样品 & 摩尔比 & & & & \\
\hline & 葡萄糖 & 半乳糖 & 甘露糖 & 阿拉伯糖 & 岩藻糖 \\
\hline $\mathrm{PNW}^{\mathrm{a}}{ }^{\mathrm{a}}$ & 1.00 & 2.36 & & & \\
\hline PNW $1^{\text {b }}$ & 1.00 & 2.03 & 2.62 & & \\
\hline $\mathrm{PNW}^{\mathrm{c}}$ & & & + & & \\
\hline $\mathrm{PNW}^{\mathrm{d}}$ & & & 5.87 & 1.00 & 1.19 \\
\hline $\mathrm{PNM}^{\mathrm{a}}$ & 3.61 & 1.00 & & & \\
\hline $\mathrm{PNM}^{\mathrm{b}}$ & 3.32 & 1.00 & 1.87 & & \\
\hline $\mathrm{PNM}^{\mathrm{c}}$ & & & + & & \\
\hline $\mathrm{PNM}^{\mathrm{d}}$ & 5.96 & & 2.74 & 1.00 & 0.96 \\
\hline
\end{tabular}

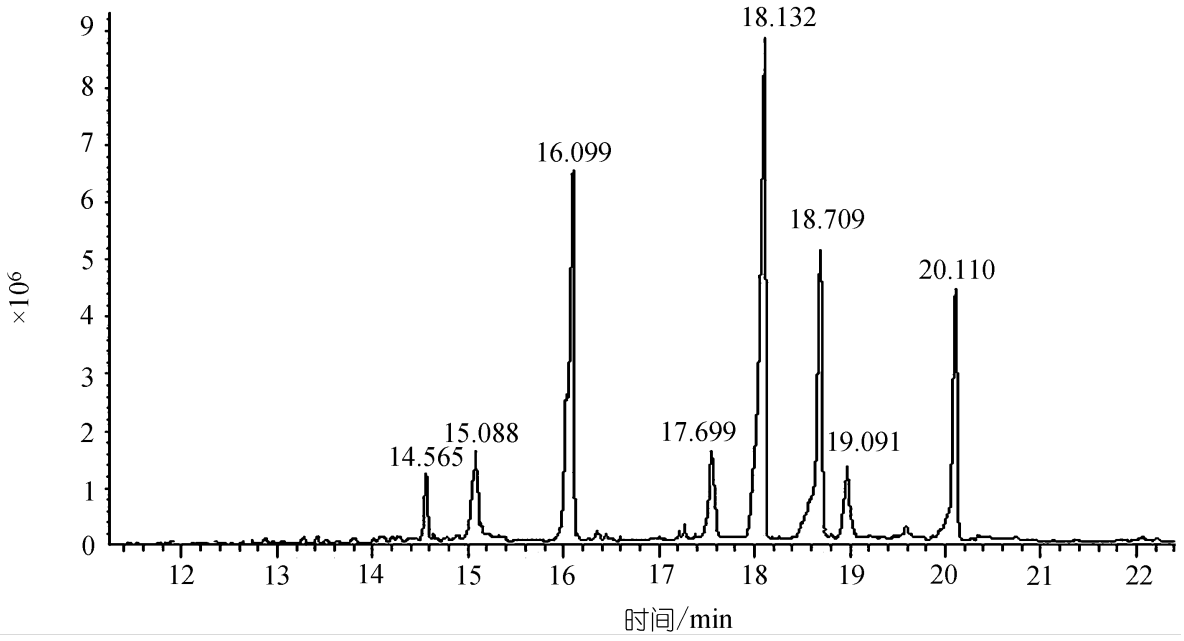

图 1 PNW1 的 GC-MS 分析总离子流图 
表 3 PNW1 和 PNM1 甲基化的 GC-MS 分析

\begin{tabular}{|c|c|c|c|}
\hline \multirow{2}{*}{ 氧甲基糖醇乙酰醋 } & \multicolumn{2}{|c|}{ 摩尔百分比(\%) } & \multirow{2}{*}{ 连接键型 } \\
\hline & PNW1 & PNM1 & \\
\hline 2,3,5- $\mathrm{Me}_{3}$-Ara & 2.35 & 2.26 & Ara $(1 \rightarrow$ \\
\hline $2,3,4-\mathrm{Me}_{3}-\mathrm{Fuc}$ & 5.37 & 1.71 & Fuc $(1 \rightarrow$ \\
\hline $2,3,4,6-\mathrm{Me}_{4}-\mathrm{Glc}$ & - & 22.43 & Glc $(1 \rightarrow$ \\
\hline 2,3,4,6- $\mathrm{Me}_{4}-\mathrm{Man}$ & 19.40 & 2.83 & $\operatorname{Man}(1 \rightarrow$ \\
\hline $2,3,4-\mathrm{Me}_{3}-\mathrm{Glc}$ & 7.93 & 6.35 & 6)-Glc $(1 \rightarrow$ \\
\hline $2,3,4-\mathrm{Me}_{3}-\mathrm{Gal}$ & 31.05 & 6.05 & 6)-Gal (1 $\rightarrow$ \\
\hline 2,3,4--Me 3 -Man & 14.02 & 31.24 & 6)-Man $(1 \rightarrow$ \\
\hline 3,4-Me $2-\mathrm{Glc}$ & 4.07 & 20.81 & $2,6)$-Glc $(1 \rightarrow$ \\
\hline $3,4-\mathrm{Me}_{2}-\mathrm{Gal}$ & 11.78 & - & $2,6)-\mathrm{Gal}(1 \rightarrow$ \\
\hline
\end{tabular}

2,3,4,6- $\mathrm{Me}_{4}$-Glc=1,5-di-O-acetyl-2,3,4,6-tetra-O-methyl-glucitol, - 未检测出

${ }^{13} \mathrm{C}$ NMR 分析（图 2)，在 $\delta(97 \sim 103) \times 10^{-6}$ 处有 4 个明显的 C-1 位移. $\delta 103 \times 10^{-6}$ 处最明显的信号为 $\beta-G a 1$ 异头C-1 的化学位移. $\alpha-, \beta-$ 甘露糖的化学位移 相差很小, 在 $\delta(101 \sim 102) \times 10^{-6}$ 范围内. $\delta 97 \times 10^{-6}$ 为 $\alpha-\mathrm{Gal}$ 异头C-1 的化学位移. 未取代的C-6 在 $\delta(60 \sim 66)$ $\times 10^{-6}$ 之间, 被取代的C- 6 移动到 $\delta(68 \sim 70) \times 10^{-6}$ 范围 内. PNW1 被取代C-2 的化学位移移动到 $884.274 \times$ $10^{-6}$ 处. PNW1 有 $\alpha$ 和 $\beta$ 两种构型, 以 $\beta$ 构型为主.

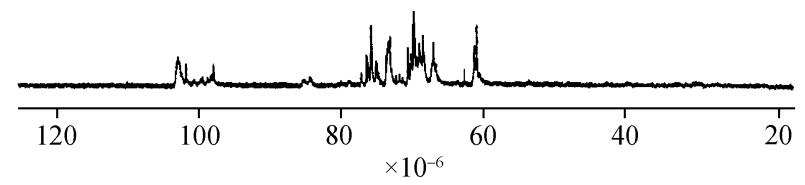

图 2 PNW1 的 ${ }^{13} \mathrm{C}$ NMR谱图

综合以上结果, PNW1 的基本结构为: $1 \rightarrow 6-\mathrm{Glc}$ 和 $1 \rightarrow 6-\mathrm{Gal}$ 构成主链, 分支点 $\mathrm{O}-2$ 连接侧链, 侧链由 $1 \rightarrow 6-$ Man 短链和 Ara, Fuc, Man 的末端构成. PNW1 的重复单元见图 3.

\subsection{PNM1 的结构分析}

PNM1 的部分酸水解结果见表 $2, P N M 1^{\mathrm{a}}$ 和PNM1 ${ }^{\mathrm{b}}$ 中检出葡萄糖和半乳糖，它们构成PNM1 的骨架结构. $\mathrm{PNM}^{\mathrm{d}}$ 中检出大量的甘露糖, 说明支链主要由甘露糖 构成. PNM1 可以被高碘酸氧化, 消耗 $0.274 \mathrm{~mol}$ 高碘 酸, 产生 $0.121 \mathrm{~mol}$ 甲酸. Smith 降解结果显示, 只有 甘油产生, 没有检测出赤藓醇和糖残基, 说明PNM1 含有的糖苷键类型均可被高碘酸氧化.

PNM1 的甲基化结果见图 4 和表 3. GC 共检测出 8 个峰, 经分析分别为 2,3,5-三-O-甲基-阿拉伯糖醇, 2,3,4-三-O-甲基-岩藻糖醇，2,3,4,6-四-O-甲基-葡萄糖 醇，2,3,4,6-四-O-甲基-甘露醇，2,3,4-三-O-甲基-葡萄 糖醇，2,3,4-三-O-甲基-甘露醇，2,3,4-三-O-甲基-半乳 糖醇, 3,4-二-O-甲基-葡萄糖醇. PNM1 中含有大量的 Glc $1 \rightarrow 6$, 分支点均在 Glc 的 O-2. 非还原末端和分 支点的含量很高, 说明 PNM1 为一多分支的多糖.

PNM1 的 ${ }^{13} \mathrm{C}$ NMR 结果见图 5. PNM1 有 $\alpha$ 和 $\beta$ 两种构型. $\delta 104.223 \times 10^{-6}$ 和 $\delta 104.323 \times 10^{-6}$ 分别为 $1 \rightarrow$ 和 $1 \rightarrow 2,6$ - 连接的 $\beta$-Glc 异头 C- 1 的化学位移. $\delta(101 \sim 102) \times 10^{-6}$ 间为 $\beta$-或 $\alpha$-Man 的 C-1 位移. $\delta(98 \sim$ 99) $\times 10^{-6}$ 间为 $\alpha-G a 1$ 或 $\alpha-G l c$ 的C-1 位移. PNM1 被取代 C-2 的化学位移移动到 $\delta 80.398 \times 10^{-6}$.

综合以上结果, PNM1 的基本结构为 $1 \rightarrow 6-$ Glc 和 $1 \rightarrow 6-\mathrm{Gal}$ 构成主链, Glc 的 $\mathrm{O}-2$ 为分支点连接侧链, 侧 链由 1 $\rightarrow$ 6-Man 短链和 Ara, Fuc, Glc 和 Man 的末端 构成. PNM1 的重复单元见图 6.

\section{4 抗肿瘤活性}

小鼠移植Sarcoma-180 肉瘤模型检测 PNW1 和 PNM1 的抗肿瘤效果 ${ }^{[21]}$. 结果显示, 实验剂量为 400 $\mathrm{mg} / \mathrm{kg}$ 时PNW1 的抑瘤率高达 $74.70 \%$, PNM1 的抑瘤 率也很高, 为 $55.84 \%$ (图 7).

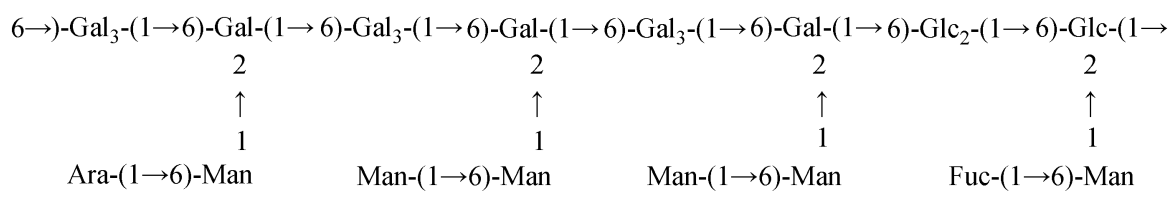

图 3 PNW1 的重复单元 


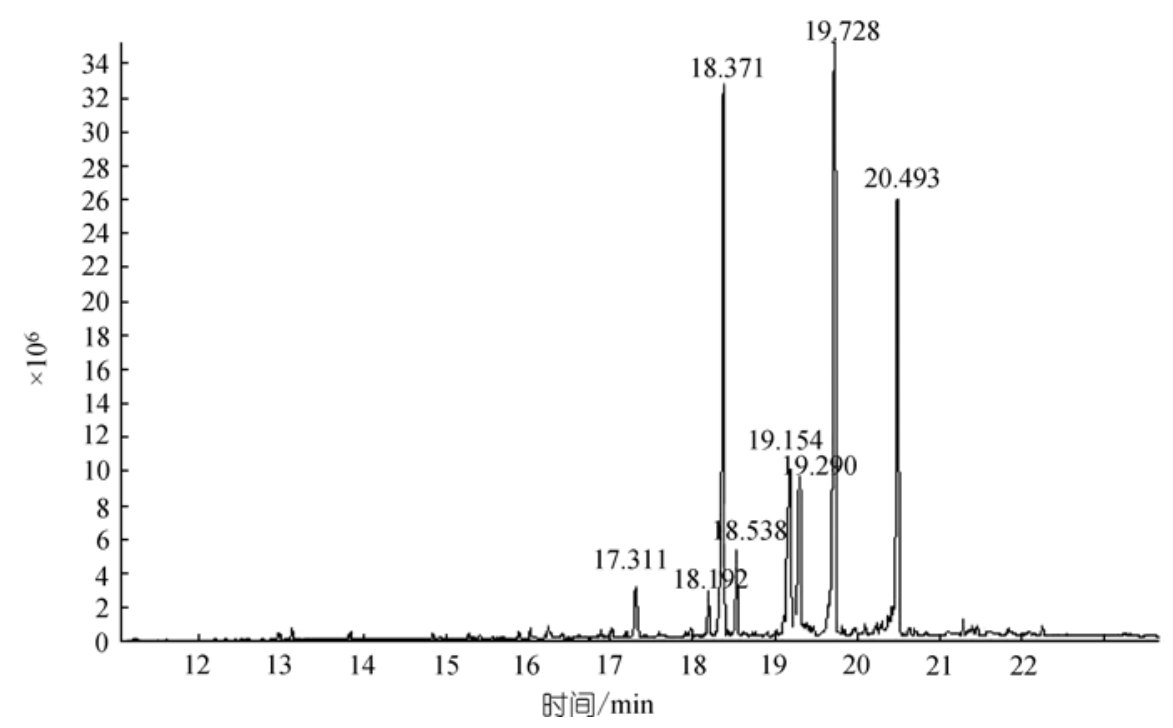

图 4 PNM1 的 GC-MS 分析总离子流图

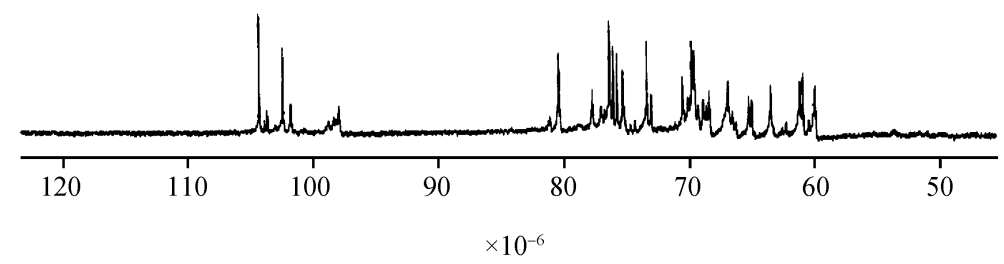

图 5 PNM1 的 ${ }^{13} \mathrm{C}$ NMR谱图

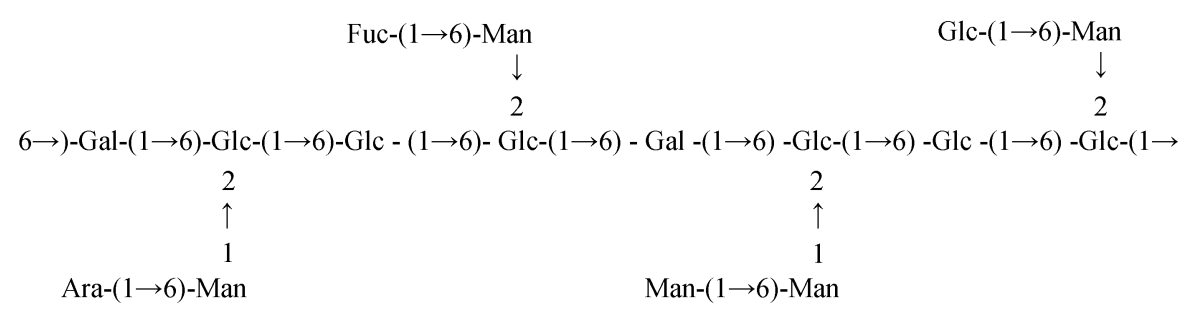

图 6 PNW1 的重复单元

\section{3 讨论}

已经报道, 真菌多糖和糖-蛋白复合物具有抑制 Sarcoma 180 瘤细胞生长的作用 ${ }^{[22]}$. 在亚洲一些国家, 如中国和日本，已经把一些有活性的真菌多糖开发 成抗肿瘤药物并广泛应用 ${ }^{[23]}$. 本实验组已经发现黑 盖木层孔菌发酵液多糖具有明显的免疫和抗肿瘤活 性, 本文结果显示菌丝体多糖的抗肿瘤效果是由于
发酵液多糖.

多糖的抗肿瘤活性与其结构是相关的. 具有抗 肿瘤活性的真菌多糖大多为葡聚糖, 也有一些杂多 糖. 香菇多糖 Lentinan 和裂褶菌多糖 Schizophyllan 为 $\beta$-葡聚糖, 云芝多糖 PSK 为结合有蛋白的 $\beta$-葡聚糖. 而 PSPC 为多糖-蛋白复合物, 由半乳糖、葡萄糖、甘 露糖、阿拉伯糖、木糖、鼠李糖和岩藻糖及蛋白构 


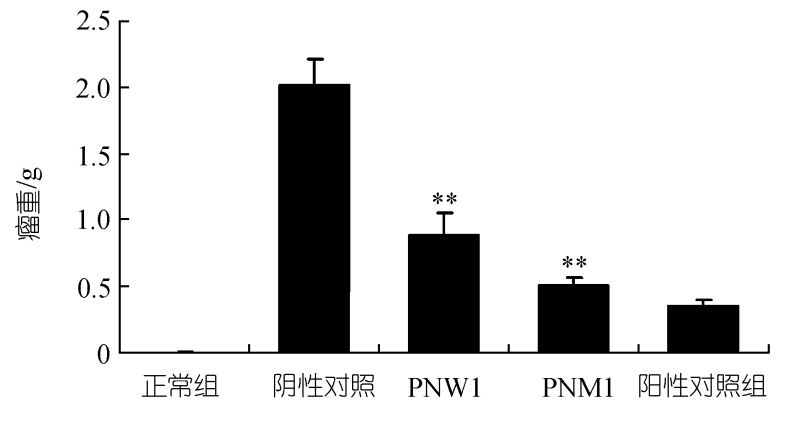

组

图 7 PNW1 和 PNM1 的抗肿瘤效果

模型组、阳性对照组和多糖组均植入 Sarcoma 180 细胞, 正常组不 接种瘤细胞, $* * P<0.01$

成 $[24,25]$. 多糖的分子量对活性也有较大的影响. 一般
认为, 多糖的分子量高抗肿瘤活性明显. 但是从松山 灵芝(Ganoderma tsugae)子实体中提取的一种杂多糖 有很好的抗肿瘤效果, 分子量仅 $10 \mathrm{kD}$, 糖组成为半乳 糖、葡萄糖、甘露糖、和岩藻糖; 菌丝体中提取的高 活性多糖为蛋白多糖, 分子量为 $10 \mathrm{kD}^{[26,27]}$. 本实验从 黑盖木层孔菌菌丝体和发酵液中分别纯化出活性多糖 PNW1 和 PNM1, 它们有相似的分子量, 分别为 33 $\mathrm{kD}, 29 \mathrm{kD}$, 主要由葡萄糖、半乳糖、甘露糖、阿拉伯 糖和岩藻糖构成. PNW1 中半乳糖的含量很高, 达到 $42.9 \%$; 而PNM1 中匍萄糖为主 $(51.4 \%)$. 这两种多糖 的结构也不同, 本实验确定了它们的结构重复单元.

黑盖木层孔菌具有抗肿瘤活性, 但是其他生物 活性还没有被研究，具有很好的研究前景. 其生物活 性和病理效果还有待深入研究.

\section{参考文献}

1 Ooi V E C, Liu F. Immunomodulation and anticancer activity of polysaccharide protein complexes. Curr Med Chem, 2000, 7: $715-729$

2 Iagher F, Reicher F, Ganter J L M S. Structural and rheological properties of polysaccharides from mango (Mangifera indica L.) pulp. Int J Biol Macromol, 2002, 31: 9-17 $\underline{\text { [DOI] }}$

3 Saima Y, Das A K, Sarkar K K, et al. An antitumor pectic polysaccharide from Feronia limonia. Int J Biol Macromol, 2000, 27: $333-335$ [DOI]

4 Yang Y, Zhang J S, Liu Y F, et al. Structural elucidation of a 3-O-methyl-D-galactose-containing neutral polysaccharide from the bodies of Phellinus ignirius. Carbohydr Res, 2007, 342: 1063-1070[DOI]

5 Dong Q, Yao J, Fang J N, et al. Structural characterization and immunological activity of two cold-water extractable polysaccharides from Cistanche deserticola Y.C.Ma. Carbohydr Res, 2007, 342: 1343-1349[DOI]

6 Krcmar P, Novotny C, Marais M F, et al. Structure of extracellular polysaccharide produced by lignin-degrading fungus Phlebia radiata in liquid culture. Int J Biol Macromol, 1999, 24: 61-64[ㅁO]

7 李霞, 田文敏, 黄明, 等。黑盖木层孔菌胞外多糖的提取及活性研究. 中国生物工程杂志, 2007, 27(4): 50一 53

8 Ajith T A, Janardhanan K K. Cytotoxic and antitumor activities of a polypore macrofungus, Phellinus rimosus (Berk) Pilat. J Ethnopharmacol, 2003, 84: 157-162[DOI]

9 Shon M Y, Kim T H, Sung N J. Antioxidants and free radical scavenging of Phellinus. baumii (Phellinus of Hymenochaetaceae) extracts. Food Chem, 2003, 82: 593-597[DOD]

10 Baea J S, Janga K H, Yim H N, et al. Polysaccharides isolated from Phellinus gilvus inhibit melanoma growth in mice. Cancer Lett, 2005, 218: 43-52[DOI]

11 Kim H M, Han S B, Oh G T, et al. Stimulation of humoral and cell meditated immunity by polysaccharide from mushroom Phellinus linteus. Int Immunopharmacol, 1996, 18: 295-303[DOI]

12 Han S B, Lee C W, Jeon Y J, et al. The inhibitory effect of polysaccharides isolated from Phellinus linteus on tumor growth and metastasis. Immunopharmacol, 1999, 41: 157-164 $\underline{\text { [DOI] }}$

13 Li G, Kim D H, Kim T D, et al. Protein bound polysaccharide from Phellinus linteus induces $\mathrm{G}_{2} \mathrm{M}_{\text {phase }}$ arrest and apoptosis in SW480 human colon cancer cells. Cancer Lett, 2004, 216: 175-181 [DOI]

14 魏运安, 方积年. 高效凝胶色谱法测定多糖纯度及分子量. 药学学报, 1989, 24(7): 532-536 Brock T D. Biotechnology: a textbook of industrial microbiology. USA: Sinauer Associates Inc, 1990. 91—193 
16 Blumenkrantz N, Asboe-Hansen G. New method for quantitative determination of uronic acid. Anal Biochem, 1973, 54: $484-489 \underline{[\mathrm{DOI}]}$

17 Oades J M. Gas - liquid chromatography of alditol acetates and its application to the analysis of sugars in complex hydrolysates. J Chromat, 1967, 28: 246-252[DOI]

18 Johnes T M, Albersheim P. A gas chromatographic method for the determination of aldose and uronic acid constituents of plant cell wall polysaccharide. Plant Physiol, 1972, 49: 926-936

19 Paulnock D M. Macrophage activation by T-cells. Curr Opin Immunol, 1992, 4: 344-349 [DOD]

20 Needs P W, Selvendran R R, Selvendran R R. Avoiding oxidative degradation during sodium hydroxide/methyl iodide-mediated carbohydrate methylation in dimethyl sulfoxide. Carbohydr Res, 1993, 245: 1-10 $\underline{\text { [DOI] }}$

21 Ikekawa T, Uehara N, Maeda Y, et al. Antitumor activity of aqueous extracts of edible mushrooms. Cancer Res, 1969, 29: 734-735

22 Jong S C, Donovick R. Antitumor and antiviral substances produced from fungi. Adv Appl Microbiol, 1989, 34: 183-262[DOI]

23 Fukushima M. The over dose of drugs in Japan. Nature, 1989, 342: 850-851 [DOI]

24 Liu F, Ooi V E C, Chang S T. Anti-tumour components of the culture filtrates from Tricholoma sp. World J Microbiol Biotech, 1995, 11: $486-490 \underline{\text { [DOI] }}$

25 Liu F, Fung M C, Ooi V E C, et al. Analysis of immunomodulating cytokine mRNAs in the mouse induced by mushroom polysaccharides. Life Sci, 1996, 58: 1795-1803 [DOI]

26 Mizuno T, Saito H, Nishitoba T, et al. Antitumor active substrances from mushrooms. Food Rev Int, 1995, 11: 23-61

27 Zhang J, Wang G, Li H, et al. Antitumor active protein-containing glycans from the Chinese mushroom Songshan Lingzhi, Ganoderma tsugae mycelium. Biosci Biotech Biochem, 1994, 58: 1202-1205 